\title{
The efficacy of tapentadol prolonged release in the treatment of mixed cancer pain
}

\author{
Elwira Góraj
}

Pain can be experienced at every stage of cancer and its treatment (50\% to 90\%); about 19-39.1\% of patients suffer from neuropathic pain and $75 \%$ from mixed pain. Due to different treatment strategy a proper diagnosis of pain is of high importance. Opioids with a pure agonist effect are used to treat moderate and severe nociceptive pain and adjuvant drugs in the first line treatment of neuropathic pain.

Tapentadol is the only centrally acting opioid that combines two mechanisms of action - mu-opioid receptor (MO$\mathrm{R}$ )-agonist and norepinephrine reuptake inhibitor (NRI) activities. This specificity of action particularly predisposes tapentadol for use in mixed pain as well as in the therapy of various pain syndromes, both in the mechanism of receptor and neuropathic pain. The indication for the use of tapentadol is the treatment of chronic pain of high severity in adults, which can be properly controlled only after the use of opioid analgesics.

In experimental and clinical studies, the efficacy and good safety profile of tapentadol was proofed, both in acute (somatic and visceral) and chronic pain syndromes, including neuropathic pain.

Most of the studies concern chronic non-cancer pain. In the present case, the aspect of the occurrence of mixed cancer pain in course of pancreatic cancer with coexisting chemotherapy-induced neuropathy (treated with gabapentin) is emphasized. Due to the lack of good visceral control the combined method was used with opioids i.e. fentanyl TTS and oxycodone. Neurolysis of the celiac plexus was performed, followed by followed by main opioid rotation for tapentadol PR, resulting in a reduction in basic (visceral and neuropathic pain), and a reduction in the severity of breakthrough pain episodes, which were controlled by rapid-acting transmucosal fentanyl.

NOWOTWORY J Oncol 2018; 68, 3: 146-151

Key words: cancer pain, neuropathic pain, opioids, chemotherapy-induced polyneuropathy, tapentadol PR

\section{Introduction}

Pain can be experienced at every stage of cancer and its treatment. The early implementation of appropriate analgesics and adjuvant drugs in accordance with recommendations of WHO analgesic ladder and the guidelines of panel of expert scientific societies allows to sufficiently control pain in most cases. In moderate to severe pain, opioids are used. When choosing the right opioid, one should take into account the mechanism and intensity of pain, the patient's age, metabolism and confounding factors, co-morbidities, medication, psychological status, reaction to the previously used opioid and the history of drug abuse.
Potent opioids are usually classified by affinity for opioid receptors and divided into full agonists, partial agonists and agonist-antagonists.

The most frequently used are opioids from the group of pure agonists that do not have a definite maximum dose. Opioids that are partial agonists or agonist-antagonists have so-called ceiling effect. In Poland, the most commonly used partial agonist is buprenorphine (a partial agonist of the $\mu$ and $\delta$ opioid receptors and the $\mathrm{k}$ receptor antagonist) which, when used in therapeutic doses, acts like a pure agonist without a ceiling effect for analgesia. 
In contrast to other opioids, buprenorphine has a beneficial effect from the safety perspective of therapy (e.g. the risk of opioid overdose), a ceiling effect for respiratory depression. The maximum recommended dose of transdermal buprenorphine is $140 \mathrm{mcg} / \mathrm{h}$.

Buprenorphine is a partial agonist with low intrinsic activity. Opioids included in agonist-antagonists (butorphanol, nalbuphine, pentazocine) act agonistically on one of the receptors (mainly $\mu$ ) and antagonistically on others. None of these is currently used in the treatment of acute and chronic pain [1, 2].

The pure mu-opioid receptor (MOR) agonists are classified according to the number of $\mathrm{Ki}$, determining the degree of affinity for the receptor (binding strength).

Opioids were divided into three groups due to the K value:

- $\mathrm{K}(\mathrm{i})>100 \mathrm{nM}$ tramadol, codeine, meperidine, propoxyphene, pentazocine;

- $\mathrm{K}(\mathrm{i})=1-100 \mathrm{nM}$ hydrocodone, oxycodone, diphenoxylate, alfentanil, methadone, nalbuphine, fentanyl, morphine;

- $\mathrm{K}(\mathrm{i})<1 \mathrm{nM}$ butorphanol, levorphanol, oxymorphone, hydromorphone, buprenorphine, sufentanil [3].

The smaller the Ki value, the greater the affinity for the $\mu$ receptor.

The most common in the treatment of cancer pain are drugs with a pure agonist action in relation to the known types of opioid receptors (strong affinity for $\mu$ receptors and weak in relation to the $\mathrm{K}$ and $\delta$ receptor).

Morphine and fentanyl are characterized by a similar profile of interaction with major classes of receptors. Methadone is characterized by a strong interaction of the $\mu$ and $\delta$ receptors. Oxycodone shows the strongest affinity for $\kappa$ receptors $[1,2,4,5]$.

The duration of action may be pharmacologically determined (receptor binding mode), e.g. levorphanol and methadone, or pharmaceutically (tablet structure, patch) in sustained release formulations such as SR morphine, oxycodone, tapentadol PR, transdermal fentanyl, transdermal buprenorphine.

The side effects of opioids, which are most often clinically observed: nausea, sedation, constipation, respiratory depression, are the effect of activation of opioid receptors. Less common side effects, such as myoclonus, hallucinations and disorientation, are not reversed after administration of pure antagonists. Treatment of these symptoms requires a reduction of the opioid dose or a change of preparation (rotation). Quetiapine, haloperidol, olanzapine and chlorpromazine are also used in the treatment of hallucinations and myoclonus. Symptoms usually increase proportionally to the opioid dose, and partial or total rotation is usually necessary. Deciding on rotation, metabolic causes of symptoms, such as hyponatremia, hypercalcemia or metastases to CNS should be excluded.
Nausea and vomiting may occur in $10-40 \%$ of patients at the initial stage of treatment. Most patients develop tolerance to this symptom, some require periodic administration of antiemetics.

Constipation remains a persistent problem during the treatment with opioids. Therefore, the disorder does not develop the phenomenon of tolerance. In many cases of opioid-induced constipation an effective solution is partial or complete change to a preparation with a proven lower risk of this side effect. The solution to the problem may be the conversion of a hydrophilic opioid to a lipophilic opioid, e.g. buprenorphine, fentanyl. In persistent cases, a combination of an agonist and antagonist (acting locally at intestinal level) should be used - oxycodone with naloxone. The lack of an opioid with a favorable profile of side effects encourages the search for new analgesics, preferably with a different mechanism of action and high analgesic efficacy, while not exacerbating unpleasant side effects [1, 6-8].

\section{Tapentadol}

Tapentadol is the latest opioid preparation introduced for the treatment of chronic pain as a strong opioid of the third step of analgesic ladder. Originally, a fast-acting form was created, and then a long-acting (prolonged release, PR) formula was developed. Currently available in Poland doses of tapentadol PR are $50 \mathrm{mg}, 100 \mathrm{mg}, 150 \mathrm{mg}, 200 \mathrm{mg}$ and $250 \mathrm{mg}$.

Tapentadol, a centrally acting opioid with strong analgesic effect due to the dual mechanism of action contained in one molecule. The binding strength is $\mathrm{K}(\mathrm{i}) 0.1 \mathrm{M}$. It is a mu-receptor agonist that simultaneously blocks the reuptake of norepinephrine (NRI - noradrenaline reuptake inhibitor). The affinity for the $\mu$-receptor is 50 times weaker than in the case of morphine $[1,9,10]$.

The affinity for the other opioid receptors (opioid receptor - k, opioid receptor $\delta$, ORL 1 - opioid receptor-like receptor) is lower than for the MOR receptor. The degree of inhibition of serotonin reuptake is considered to be negligible and neglected in the characteristics of drug action [10].

It is highly probable (studies on animal models) that in cases of acute pain an agonistic activity on MOR receptor predominates in relation to the inhibitory effect of NRI.

Similarly, rats and mice in vivo experiments show a strong effect of tapentadol (which is $2-3$ times weaker than morphine) in acute nociceptive pain. It is believed that the NRI mechanism synergistically contributes to the overall analgesic effect of tapentadol. The NA inhibitory effect is probably more important in chronic pain [9].

In relation to oral morphine, the conversion rate 1:3.3 is most commonly used [1].

Tapentadol is mainly metabolized in the liver, in the glucuronidation process, to inactive glucuronides or sulphates, primarily to glucuronyl-O-tapentadol (55\%). Tapentadol and 
its metabolites are mainly excreted by the kidneys (99\%) and $1 \%$ of the drug is excreted in the faeces. It is very limited metabolism with the involvement of cytochrome P450 isoenzymes, which limits the potential of interactions of tapentadol with other drugs. It is most metabolized by CYP2C9, CYP2C19, CYP2D6. Tapentadol does not inhibit or excite any of the CYP isoforms [11].

Tapentadol exhibits in vivo efficacy in heat-induced hyperalgesia models with a minimum effective dose ED50. Its action in diabetic neuropathy is well-established.

It is believed that the total analgesic effect in cases of neuropathic pain results from the simultaneous activation of both mechanisms: MOR and NRI.

The antinociceptive effect of tapentadol is partially reversed by naloxone (the opioid portion of MOR), and the effect of the non-nociceptive mechanism can be reduced by administration of yohimbine, a a (alpha) 2-adrenergic blocker, affecting the sympathetic nervous system [9].

Inhibition of NA reuptake enhances the downstream pain-inhibiting pathway through a2-adrenergic receptors [10].

The moderate affinity to MOR opioid receptor and the demonstrated opioid-saving effect suggest that tapentadol should elicit less side effects associated with the use of opioid drugs compared to classical agonists. Indeed, in the comparative studies of morphine-tapentadol, oxycodone-tapentadol, fentanyl-tapentadol, it was shown that tapentadol to a lesser extent induces nausea and vomiting, as well as the duration of these symptoms is significantly shorter. Adverse symptoms occur at higher doses and the threshold for triggering symptoms is 100 times higher for tapentadol than for morphine.

Tapentadol has a weaker inhibitory effect on intestinal peristalsis than the equivalent dose of morphine (assessed by means of intestinal transit time labeled with carbon) [12]. A lower risk of causing adverse reactions was confirmed in the Merker study (2012). In the meta-analysis, the incidence of vomiting and constipation was significantly lower in the group of patients receiving tapentadol compared to the oxycodone group [7], while tapentadol to a greater extent promotes the occurrence of dryness in the mouth. Similar data were obtained by Mercadante, investigating the efficacy and tolerance of the drug among patients with cancer pain who had never received opioids (so-called opioid naive) [13].

According to available evidence, tapentadol inhibits the uptake of serotonin to a negligible extent, so it also has no side effects (constipation, nausea, vomiting, diarrhea) resulting from an increase in serotonin in the central and intestinal nervous system [10].

\section{Neuropathic pain in the course of cancer}

The latest definition of neuropathic pain accepted by the Committee on the Taxonomy of the International Asso- ciation for the Study of Pain (IASP) states that neuropathic pain is pain caused by injury or illness of the somatosensory part of the nervous system. It is estimated that 50\% to $90 \%$ of cancer patients experience pain during their lifetime [14]. Among them 19-39.1\% suffer from neuropathic pain [15]. Early diagnosis of symptoms is particularly important due to a different treatment strategy as compare to nociceptive pain. Among numerous causes of neuropathic pain in the course of neoplastic disease, factors related to tumor growth as well as treatment methods are mentioned, for example radiation-induced plexopathy (RIP) or chemotherapy-induced peripheral polyneuropathy (CIPN). There are less frequent reports of neuropathic pain in the abdominal cavity, which is why they are not recognized early enough. Mechanical causes include biliary obstruction, impaired intestinal obstruction, organ perforation, advanced colorectal cancer, intraperitoneal chemotherapy. In cases of sympathetic plexus disorders, bladder dysfunction or orthostatic hypotension appear [14-16].

Pain in the course of pancreatic cancer may be visceral, somatic or neuropathic. It results from tissue damage, local inflammation, infiltration or mechanical obstacles. The signals are conducted by sympathetic fibers constituting the innervation of the visceral plexus from the level of Th12-L2 [17]. Feeling of paroxysmal stabbing or burning pain is sometimes confused with incidents of breakthrough pain. Pain intensifies at night, disrupts daytime activity and hinders basic functions such as dressing, washing, combing and rest at night. The relationship between clinically occurring symptoms and etiology is not always clear [14].

\section{Case report}

The patient aged 62 was treated for 6 months due to inoperable pancreatic cancer (adenocarcinoma ductale pancreatis $\mathrm{G} 2$ ) and received palliative chemotherapy. The patient was given 12 courses according to the FOLFIRNOX protocol: fluorouracil + calcium folinate + oxaliplatin + irinotecan. There were co-existing problems: arterial hypertension, hyperlipidemia, history of two prior myocardial infarctions (2011 and 2014) and coronary angioplasty. During the subsequent courses of chemotherapy, the patient had grade I neutropenia.

Common side effects of FOLFIRNOX are: neutropenia, neutropenic fever, thrombocytopenia, sensory neuropathy (platinum derivatives), diarrhea.

The neoplastic lesion was located in the head of the pancreas. The patient reported epigastric pain radiating to the spine at Th10-12. In addition, there was a feeling of numbness and tingling in the hands and feet (greater in feet). Symptoms began to intensify after the eighth cycle of chemotherapy. It was a mixed type of pain, with a distinct neuropathic component, described as pulsating, squeezing and radiating to the back. The patient was diagnosed with 
two neuropathic pain: the first one as a pancreatic component, the second - peripheral polyneuropathy. Previous pharmacological treatment didn't sufficiently controlled the pain. The patient assessed his present pain intensity in the VAS scale (0-100) on 80-90, with periodic exacerbations up to 100 .

The initial diagnosis was: visceral pain within the abdominal cavity in the course of pancreatic cancer, comorbid with pain of the nature of neuropathy in the hands and feet, with a feeling of numbness and tingling (complication after chemotherapy). The patient was referred by an oncologist to be qualified for interventional pain management (neurolysis of celiac plexus).

ECOG performance status was 2. The results of lab tests confirmed adequate liver and kidneys functions. Clinical symptoms of pre-cachexia have been observed, risk related to the nutritional status NRS (nutritional risk score) $2002=2$.

Surgery was postponed due to the lack of sufficient assessment of the cardiac output - ejection fraction (EF). Heart failure is diagnosed below $50 \%$, whereas with an $\mathrm{EF}$ of $>45 \%$, there is a risk that the patient will have a fall in blood pressure following the expansion of the abdominal vascular bed.

The patient was admitted to establish analgesia. Drugs previously used were: oxycodone $60 \mathrm{mg}$ b.i.d., fentanyl transdermal system $150 \mathrm{mcg} / \mathrm{h}$ every 3 days, morphine IR 20 mg PRN. Other medicines used: low molecular weight heparin $40 \mathrm{mg} / 0.41 \mathrm{amp}$. q.d. s.c., spironolactone 25 mg every other day, furosemide $40 \mathrm{mg}$ q.d., metoprolol 25 mg q.d., pancreatin 25 thousand $U$ q.d. with a main meal, alprazolam 0,25 mcg b.i.d., ketoprofen duo1 table PRN, ketoprofen forte $100 \mathrm{mg}$ b.i.d.

To extent the diagnosis, a CT scan of the abdominal cavity was performed, which showed an advanced local cancer. In the area around the head of the pancreas, a solid-fluid lesion with approximate size $83 \mathrm{~mm} \times 87 \mathrm{~mm} \times 70 \mathrm{~mm}$ was visible. The lesion adhered to the aorta and duodenum and infiltrated the initial segments of the celiac trunk and upper mesenteric artery. The Wirsung duct distal to the lesion was widened to approx. $7 \mathrm{~mm}$. The body and tail of the pancreas were normal. Enlarged peripancreatic lymph nodes and several lymph nodes along the aorta and inferior vena cava up to $36 \mathrm{~mm} \times 25 \mathrm{~mm}$ were observed. The liver was normal, with no focal lesions. Bones without visible secondary lesions.

The dose of oxycodone was increased to $200 \mathrm{mg} /$ day (below the calculated equivalent dose) with rotating within 3 days by $1 / 3$ of daily dose. Fentanyl TTS was discontinued in two stages: reduction to $100 \mathrm{mcg} / \mathrm{h}$ and after another 3 days to $50 \mathrm{mcg} / \mathrm{h}$.

Slow rotation allows better tolerance of treatment change. Salvage Rescue therapy with morphine IR $20 \mathrm{mg}$ p.o. was used to titrate a requirement for strong opioid and achieve satisfactory pain control, and lactulose $3 \times 20 \mathrm{ml}$ as prevention of constipation. Gabapentin $100 \mathrm{mg}$ t.i.d. as co-analgetic was added, gradually increasing to $300 \mathrm{mg}$ t.i.d. due to the diagnosis of CIPN. A satisfactory analgesic effect was obtained, however, after 4 days the patient reported increasing constipation. Stool with a hard consistency appeared every 3-4 days, with flatulence, nausea and lack of appetite. In case of opioid-induced constipation constipation, conversion to another opioid with a lower risk of constipation should be considered. It was decided to convert a part dose of oxycodone daily dose $(200 \mathrm{mg}$ ) to oxycodone with naloxone with the ratio $1 / 3$ oxycodone with naloxone $60 \mathrm{mg} /$ day and $2 / 3$ oxycodone ( $140 \mathrm{mg} /$ day). Instead of morphine IR INN-fentanyl buccal tablet should be considered, $400 \mathrm{mcg}$ (after titration) was used to treat breakthrough pain, up to 4 times a day.

Lack of good analgesic effect, despite relatively high doses of opioid, may be caused by the increasing tolerance for a given opioid. The indication for conversion to another opioid is the development of tolerance, difficult to control adverse effects and unfavorable balance between analgesic effect and adverse effects.

Tolerance to opioids should be distinguished from the resistance of some types of pain to opioids (e.g. neuropathic pain). If the indication for rotation is the development of tolerance, or when the first opioid is used in high doses treatment with the second opioid begins from 1/2-1/4 of the calculated equivalent dose. Tables with equivalent doses are only an example (guide) of how to calculate them, and they should be approached with caution.

Due to the lack of satisfactory pain control and increasing opioid-induced constipation - after obtaining a positive echocardiography result (EF 49\%) - neurolysis of the celiac plexus was performed. Good analgesic effect was obtained.

In subsequent days, the dose of oxycodone was reduced every 3 days depending on the situation, without changing the dose of gabapentin $(3 \times 300 \mathrm{mg})$. Due to the duration of episodes of visceral pain INN-fentanyl buccal tablet $(200 \mathrm{mcg}$ ) was maintained as a rescue medicine. The patient achieved a reduction in primary pain intensity up to 50 according to VAS scale.

Nausea and periodic vomiting appeared again. Abdominal X-ray revealed partial intestinal sub-obstruction. Conservative treatment was introduced: metoclopramide i.v., spasmolytic drugs i.v., dexamethasone i.v. Due to high dose of transdermal fentanyl previously taken ( $150 \mathrm{mcg} / \mathrm{h}$ ) and lack of analgesic effect it seemed doubtful that the return to fentanyl TTS would be effective. In addition, due to peripheral edema and low molecular weight heparin taken, subcutaneous route would be associated with the formation of hematomas after injection and impaired absorption of the drug. 
Over the next 4 days, analgesia was carried out with morphine i.v. in a PCA (patient controlled analgesia) pump. The pump was equipped with a button, triggering the supply of the programmed dose of rescue medication whenever was pressed by the patient. The conversion dose: oxycodone $200 \mathrm{mg}$ = morphine $300 \mathrm{mg}$ (conversion rate 1:1.5), 1/3 of the oral dose $=100 \mathrm{mg}$ i.v. dose, which is $100 \mathrm{mg} / 24 \mathrm{~h}$; and $4.1 \mathrm{mg} / \mathrm{h}$ as a continuous infusion. In addition, a PCA $1 \mathrm{mg}$ bolus was programmed with lockout time $15 \mathrm{~min}$.

After controlling nausea and vomiting, morphine i.v. was changed to tapentadol PR p.o. The choice of opioid was guided by the nature of the discomfort (mixed pain), partial occurrence of receptor (visceral) pain and partly neuropathic pain (peripheral polyneuropathy). Tapentadol also has a much more favorable profile of gastrointestinal side effects vs other potent opioids (e.g. oxycodone, morphine). The dose of tapentadol retard $200 \mathrm{mg}$ b.i.d (less than equivalent) was included, keeping the INN-fentanyl buccal tablet $200 \mathrm{mgc}$ as a rescue medicine. After the celiac plexus neurolysis and opioid re-rotation, the intensity of the basic pain stabilized at level 40 according to the VAS scale.

Final recommendations for pain management: tapentadol retard $200 \mathrm{mg}$ b.i.d., INN-fentanyl buccal tablet $200 \mathrm{mcg} /$ dose, not more than 4 times per day, gabapentin $3 \times 200 \mathrm{mg}$ t.i.d. for slow dose reduction and withdrawal.

It should be bear in mind that neuropathic pain, after controlling the dominant visceral pain, may be subjectively perceived as stronger (it begins to dominate) and reported as "more troublesome".

\section{Discussion}

In case of neuropathic pain, the plasticity of the nervous system causes pain to feel without a noticeable pain stimulus, so-called the phenomenon of spontaneous pain, or a painful response to the stimulus that does not usually provoke pain (allodynia), or increased pain from a stimulus that usually provokes pain and (hyperalgesia).

One of the mechanisms is sensitization at the level of the spinal cord and the upper levels of the CNS. Among the inhibitory mechanisms, the descending pathways, beginning in the midbrain and the medulla are important. They can inhibit or support the transmission of pain in the spinal cord. Serotonin and noradrenaline (NA) are important neurotransmitters. A painful stimulus may initiate a reversible reaction and the release of NA binding to alpha2 adrenergic receptors $[14,15]$.

The serotonergic pathway of the descending pain inhibition system can facilitate or inhibit impulses conduction across the various 5-HT receptor subtypes. Under normal conditions, constant descending and $a 2$ adrenoceptor inhibition occurs.

At the time of nerve damage, conduction by descending path is increased. This phenomenon explains the lack of efficacy of selective serotonin reuptake inhibitor (SSRIs) in the treatment of chronic neuropathic pain. SSRIs indirectly cause simultaneous activation of inhibition as well as stimulation of 5-HT receptors [10].

Tapentadol (deprived of serotonin action), increasing the activity of descending noradrenergic pain inhibition pathways and simultaneously by activating opioid receptors inhibiting the ascending pathways of pain in the CNS, seems to be an interesting drug for the treatment of neuropathic pain syndromes [18]. The drug is slightly bound to plasma proteins, about $20 \%$, which reduces the risk of interaction in the mechanism of protein binding. Inactive metabolites also do not cause adverse drug interactions. A small degree of microsomal metabolism through CYP enzymes minimizes the risk of interaction [11].

Neurolysis of the celiac plexus is performed mainly in cases of visceral pain located in the epigastrium caused by pancreatic, gastric and liver cancer and spread to the retroperitoneal lymph nodes. It is also performed in the case of pain related to chronic pancreatitis. The best effect is obtained if the tumor is located in the tail of the pancreas.

In the majority of patients, after the neurolysis procedure, the basic pain decreases or disappears, but the breakthrough pain remains in lower intensity. It is most often similar to primary pain and has an average of 2.7 points. Patients after neurolysis achieve better pain reduction, manifested by lower opioid consumption, reduced side effects of opioids and better quality of life [19].

In patients with nausea, vomiting or dysphagia, transdermal preparations of fentanyl and buprenorphine are an alternative to opioids administered s.c. or i.v. [1, 2].

Chemotherapy-induced polyneuropathy (CIPN) is a common side effect of chemotherapy. Oxaliplatin derivatives belong to the substance with the highest induction rate of polyneuropathy. Currently no consistent caring standard is available in the treatment of CIPN. Guidelines for the treatment of neuropathic pain should be followed. The drugs of choice according to currently available studies are: gabapentin, pregabalin, duloxetine, gels with baclofen (not available in Poland), amitriptyline [20]. The dual mechanism of action of tapentadol allows for consideration of the drug in CIPN therapy. The use of tapentadol is associated with low interaction risk. A good tolerance profile facilitates titration and rotation. Further research is needed to assess whether tapentadol may be considered as monotherapy or in combination with an adjuvant in the treatment of CIPN.

\section{Summary}

About $80 \%$ of patients with advanced pancreatic cancer suffer from severe and very severe pain, which requires intensive treatment. Pain in most cases is mixed in nature. Unrecognized neuropathic pain in the course of pancreatic diseases is a frequent cause of incorrect selection of drugs. 
Achieving good pain control is one of the most important goals of palliative care. The administration of adequately high doses and the appropriate opioid (in the adjuvant setting) may be hindered by their poor tolerance and the inability to achieve an effective dose.

In case of no analgesic effect, it is recommended to replace one opioid to another (so-called rotation), in total or partial rage, adding another adjuvant drug, providing an effective method of relieving breakthrough pain (the right drug, the right dose and route of administration for a quick analgesic effect). Whenever the clinical situation requires, changing the route of drug administration from oral to parenteral s.c./i.v., TTS is recommended. Opioid pharmacology is complex, and the individual effect is the resultant of many factors. If pharmacological methods are not sufficiently effective, one should use interventional (procedure) methods of pain management $[1,2,4]$.

Neurolysis of the celiac plexus does not always completely reduce pain. It causes a significant reduction in the intensity of basic pain, helping to reduce opioid doses, reducing the intensity of side effects and improving patients quality of life. The analgesic effect lasts about 2-3 months.

The treatment does not prevent the occurrence of breakthrough pains which require separate treatment $[17,19]$.

Most cancer patients experience mixed pain (74\%) as the disease progresses. Taking into account the different mechanisms of nociceptive and neuropathic pain, $\mu$ agonists and drugs blocking the secondary uptake of NA are used in the treatment [5].

Tapentadol is the only centrally acting opioid that combines these two mechanisms of action - mi receptor agonists (MORs) and norepinephrine reuptake inhibitors (NRIs). The specificity of tapentadol makes it highly effective in the treatment of severe mixed pain as well as neuropathic pain syndromes. Its use also results in the possibility of opioid saving (opioid-sparing effect). The simultaneous action of MOR and NRI mechanism contributes to the final analgesic effect, allowing the use of lower doses to achieve a given level of analgesia [10]. The double mechanism provides effective antinociceptive action and alleviation of hyperalgesia and allodynia [9].

Tapentadol has a more favorable profile of side effects. Significantly less gastrointestinal adverse events were observed in the conducted studies compared to fentanyl, morphine and oxycodone. A favorable pharmacokinetic profile contributes to a small number of discontinuations due to poor tolerability or side effects. Because tapentadol does not show significant serotonergic activity, there is no risk of side effects typical for SSRI, including serotonin syndrome [6].

Long-term use of tapentadol has a slower effect on tolerability compared to morphine [8]. A synergistic, dual mechanism of action and the effect of saving opioids can increase the effectiveness of treatment by improving compliance (regularity of use) [8]. The efficacy of tapentadol in the treatment of mixed and neuropathic cancer pain, including CIPN, requires further investigation.

\section{Conflict of interest: none declared}

Elwira Góraj, MD, PhD

Maria Skłodowska-Curie Institute - Oncology Cancer

Department of Oncological Diagnostics and Cardio-Oncology

and Palliative Medicine

Division of Palliative Medicine

ul. Roentgena 5, 02-781 Warszawa, Poland

e-mail:elwira.goraj@coi.pl

Received: 27 Apr 2018

Accepted: 19 Jun 2018

\section{References}

1. Opioids therapy in the $21^{\text {st }}$ century. Smith HS (ed.). New York: Oxford University Press, 2013.

2. Portnoy R, Ahmed E. Principles of opioid use in cancer pain.J Clin Oncol 2014; 32: 1662-1670.

3. Volpe DA, McMahon Tobin GA, Mellon RD et al. Uniform assessment and ranking of opioid $\mu$ receptor binding constants for selected opioid drugs. Regul Toxicol Pharmacol 2011; 59: 385-390.

4. Fallon MT, Laird BJ. A systematic review of combination step III opioid therapy in cancer pain: an EPCRC opioid guideline project. Palliat Med 2011; 25: 597-603.

5. Leppert W. Rola analgetyków opioidowych w leczeniu bólu neuropatycznego u chorych na nowotwory. Medycyna Paliatywna w Praktyce 2014; 8: 81-84

6. Riemsma R, Forbes $C$, Harker J et al. Systematic review of tapentadol in chronic severe pain. Curr Med Res Opin 2011; 27: 1907-1930.

7. Merker M, Dinges $G$, Koch $T$ et al. Undesired side effects of tapentadol in comparison to oxycodone. A meta-analysis of randomized controlled comparative studies. Schmerz 2012; 26: 16-26.

8. Ahlbeck K. Opioids: a two-faced Janus. Curr Med Res Opin 2011; 27 439-448.

9. Schroder W, Tzschentke TM, Terlinden R et al. Synergistic interaction between the two mechanisms of action of tapentadol in analgesia. J Pharmacol Exp Ther 2011; 337: 312-320.

10. Kress HG. Tapentadol and its two mechanisms of action: is there a new pharmacological class of centrally-acting analgesics on the horizon? Eur J of Pain 2010; 14: 781-783.

11. Dzierżanowski T, Ciałkowska-Rysz A. Tapentadol w leczeniu przewlekłego bólu związanego z nowotworem. Medycyna Paliatywna 2016; 8: 157-163.

12. TzschentkeTM, Christoph T, Kögel B et al.(-)-(1R,2R)-3-(3-dimethylamino1-ethyl-2-methyl-propyl)-phenol hydrochloride (tapentadol $\mathrm{HCl}$ ): a novel mu-opioid receptor agonist/norepinephrine reuptake inhibitor with broad-spectrum analgesic properties. J Pharmacol Exp Ther 2007; 323: 265-276.

13. Mercadante S, Porzio G, Ferrera P et al. Tapentadol in cancer pain management: a prospective open-label study. Curr Med Res Opin 2012; 28: 1775-1779.

14. Esin E, Yalcin S. Neuropathic cancer pain: what we are dealing with? How to manage it? Onco Targets Ther 2014; 7: 599-618.

15. Bennett MI, Rayment C, Hjermstad M et al. Prevalence and aetiology of neuropathic pain in cancer patients: a systematic review. Pain 2012; 153: 359-365.

16. Fallon MT. Neuropathic pain in cancer. Br JAnaesth 2013; 111:105-111.

17. Hameed $\mathrm{M}$, Hameed $\mathrm{H}$, Erdek M. Pain management in pancreatic cancer. Cancers (Basel) 2010; 3: 43-60.

18. Schikowski A, Krings D, Schwenke K. Tapentadol prolonged release for severe chronic cancer-related pain: effectiveness, tolerability, and influence on quality of life of the patients. J Pain Res 2014; 8: 1-8. doi: 10.2147/JPR.S72150. eCollection 2015.

19. Góraj E. Zastosowanie neuroliz w leczeniu bólu nowotworowego. Medycyna Paliatywna 2012; 4: 13-23.

20. Hershman DL, Lacchetti C, Dworkin RH et al. Prevention and management of chemotherapy-induced peripheral neuropathy in survivors of adult cancers: American Society of Clinical Oncology clinical practice guideline. J Clin Oncol 2014; 32: 1941-1967. 